\title{
Optimisation de forme en aérodynamique automobile
}

\author{
Vincent Herbert ${ }^{1, a}$, Frédérique Muyl ${ }^{1}$ et Laurent Dumas ${ }^{2}$ \\ 1 PSA Peugeot Citroën, 2 route de Gisy, 78943 Vélizy Villacoublay, France \\ ${ }^{2}$ Laboratoire Jacques-Louis Lions, UPMC, 4 place Jussieu, 75005 Paris, France
}

Reçu le 25 février 2005, accepté le 21 mars 2005

\begin{abstract}
Résumé - L'objet de cet article est de présenter une méthode automatique, robuste et multicritère, d'optimisation de forme en aérodynamique automobile. Cette méthode est appliquée à l'optimisation du coefficient de traînée $\left(C_{x}\right)$ d'une forme simplifiée de véhicule et paramétrée par les angles de lunette, de diffuseur et de rétreint. Dans un premier temps, un algorithme, basé sur la méthode stochastique des algorithmes génétiques, est développé. Ces méthodes présentent l'avantage d'être globales et multicritères mais possèdent un temps de convergence prohibitif vers la solution optimale. Pour pallier ce problème, une procédure d'évaluation approchée, basée sur les réseaux de neurones, est couplée aux algorithmes génétiques. Une étude numérique est réalisée sur la forme simplifiée de véhicule précédemment décrite, dans le but d'une part, de minimiser le coefficient de traînée de la forme étudiée et d'autre part, d'estimer le gain en temps de convergence apporté par la nouvelle méthode proposée.
\end{abstract}

Mots clés : Aérodynamique / optimisation / algorithmes génétiques

\begin{abstract}
Hybrid method for aerodynamic shape optimization in automative industry shape optimization. Various global optimization methods are constructed in order to be able to tackle realistic drag reduction problems in the automotive industry. All the methods consist in improving classical genetic algorithms, by coupling them with a fast but approximated evaluation process. The efficiency of these methods is shown, fisrt on analytical test functions, then on a drag reduction problem where the computational time of a GA is highly reduced.
\end{abstract}

Key words: Genetic algorithms / neural networks / CFD / drag coefficient

\section{Introduction}

Le travail présenté dans cet article porte sur l'optimisation de forme en aérodynamique automobile. Dans le cycle de conception d'un véhicule, l'optimisation des performances aérodynamiques est souvent un travail expérimental et empirique. L'objectif de ce travail est de proposer une méthode numérique d'optimisation capable de déterminer automatiquement la forme optimale d'une partie d'un véhicule ou du véhicule complet. La fonction de coût peut être différente en fonction de la prestation recherchée : traînée pour la réduction de $\mathrm{CO}_{2}$ émis, portance pour l'amélioration de la sécurité active et passive. La méthode développée doit également être automatique, robuste et multi-critère afin d'ouvrir la voie vers des méthodes de conception multidisciplinaire.

Une des premières tentatives d'application de cette méthode dans le domaine automobile a été présentée au congrès AMIF en 2002 et publiée en 2004 par les

${ }^{a}$ Auteur correspondant : vincent.herbert@mpsa.com présents auteurs (voir [1]). Dans cet article, un principe d'accélération de convergence des algorithmes génétiques est incorporé en couplant, de manière itérative, ces derniers avec une méthode de recherche locale (méthode BFGS).

L'intérêt d'un tel couplage a été particulièrement mis en évidence en terme de vitesse de convergence dans le cas de la recherche du minimum global de plusieurs fonctions tests analytiques. Cependant, le gain observé pour l'optimisation d'une forme automobile simplifiée s'est avéré moindre en raison de l'évaluation trop approximative par différences finies du gradient de la fonction coût. Les nouvelles méthodes d'optimisation présentées dans cet article ont pour objectif de pallier ce manque de robustesse tout en restant suffisamment rapides en terme de convergence vers l'optimum global.

Après une description à la section 2 des méthodes d'optimisation globales utilisées, leur efficacité est validée sur des fonctions analytiques à la section 3 , puis sur un cas d'optimisation aérodynamique 3D d'une forme simplifiée de véhicule à la section 4 . 


\section{Méthodes d'optimisation globale}

De nouvelles méthodes d'optimisation globale et automatique (dénommées hybrides) sont introduites dans cette section. Chacune d'elles repose sur un principe d'accélération de la méthode classique des algorithmes génétiques (AG).

Les algorithmes génétiques sont des procédures d'exploration et d'exploitation fondées sur les mécanismes de la sélection naturelle et de la génétique. Ils utilisent le principe de survie de l'individu le mieux adapté, mais aussi l'échange d'informations pseudo-aléatoires pour favoriser l'exploration (Goldberg [3]). Ces algorithmes ont l'avantage d'être multi-critères, parallélisables et de converger vers le minimum global de la fonctionnelle étudiée. Cependant, leur convergence est lente et nécessite un nombre important d'évaluations de populations.

Une manière d'améliorer la vitesse de convergence des algorithmes génétiques lorsque l'évaluation de la fonction à minimiser $x \mapsto J(x)$ est coûteuse en temps de calcul, consiste à utiliser un principe d'évaluation approchée $x \mapsto$ $\tilde{J}(x)$ de plus en plus précis de la fonction exacte. C'est ainsi que Montana et al. [6], Giannakoglou [2] ont accéléré la méthode des AG seuls par l'utilisation de réseaux de neurones fixes.

Dans le cadre de ces travaux, nous avons utilisé un mode d'évaluation approchée s'appuyant sur les réseaux de neurones de type RBF (Radial Basis Function). Ces réseaux nécessitent un choix de paramètres qui conditionnent la qualité de l'approximation de la fonction et, par conséquent, la qualité de la convergence de l'algorithme d'optimisation vers l'optimum à un niveau souhaité. L'utilisation des réseaux de neurones dans ce type de couplage se fait au niveau de la phase d'évaluation des AG. L'algorithme de couplage proposé (dénommé AGA) est décrit dans le cadre ci-après.

À l'étape 1, la construction des réseaux de neurones s'inspire principalement des travaux de Giannakoglou [2]. La construction d'un réseau de neurones comporte un grand nombre de paramètres influents sur l'interpolation créée. Giannakoglou fixe les paramètres principaux des réseaux de neurones après avoir fait une étude préalable sur des fonctions analytiques. Ce choix peut limiter la précision de l'interpolation lorsque l'algorithme est appliqué à un problème physique. Nous proposons, pour éviter de fixer a priori un choix de paramètres, d'implanter un algorithme génétique, dit secondaire, pour chaque construction d'interpolation. Cet algorithme secondaire a l'avantage de mieux s'adapter à l'évolution de la base de données et de s'appliquer sur une large gamme de problèmes physiques.

Lors de l'étape 2, toute la population est pré-évaluée en utilisant le réseau local de RBF, définie par l'algorithme secondaire, et entraîné pour chaque individu par les $n_{\mathrm{c}}$ plus proches voisins contenus dans la base de données. Le réseau utilisé est en effet dit local, car un réseau est construit de manière indépendante pour chaque individu de la population à évaluer.

Mode de couplage entre les algorithmes génétiques et
les réseaux de neurones (AGA).
- Initialisation : La population initiale est choisie
aléatoirement.
- Étape $1: N_{\text {gen }}$ générations d'AG évoluent pour les-
quelles le processus d'évaluation est exact.
L'ensemble des calculs exacts ainsi effectués constitue
une base de données utiles pour la construction des
réseaux de neurones.
- Étape $2:$ À partir de la génération $N_{\text {gen }}+1$, chaque
évaluation de la fonction coût de la phase d'évaluation
de l'AG en cours est effectuée par construction d'un
réseau de neurones.
- Étape $3:$ Évaluation exacte d'une partie de la po-
pulation choisie.
- Étape 4 : On répète les étapes 2 et 3 jusqu'à conver-
gence. À chaque génération, la base de données est
mise à jour avec les calculs exacts effectués lors de
l'étape 3 pour les constructions suivantes de réseaux.

Après cette étape de pré-évaluation, un certain nombre d'individus sont sélectionnés pour être évalués exactement : les meilleurs individus obtenus par le mode d'interpolation ainsi qu'un individu pris au hasard de la population courante. Ce dernier individu permet un enrichissement de la base pour la construction des futures interpolations.

\section{Validation des méthodes d'optimisation pour des cas analytiques}

Les méthodes d'optimisation présentées sont testées et validées sur deux fonctions tests bien connues, ayant la caractéristique principale de posséder de nombreux minima locaux mais un seul minimum global. Il s'agit de la fonction de Rastrigin à 3 paramètres et de la fonction de Griewank à 30 paramètres :

$$
\begin{gathered}
\operatorname{Rast}(x)=\sum_{i=1}^{3}\left(x_{i}^{2}-\cos \left(18 x_{i}\right)\right)+3 \\
x \in[-1 ; 1]^{3} \\
\operatorname{Griew}(x)=\sum_{i=1}^{30} \frac{x_{i}^{2}}{50}-\prod_{i=1}^{30} \cos \left(\frac{x_{i}}{\sqrt{i}}\right) \\
x \in[-10 ; 10]^{30}
\end{gathered}
$$

La minimisation de ces deux fonctions est résolue dans un premier temps par la méthode des algorithmes génétiques avec des paramètres (taille de population, nombre de générations, type de croisement et mutation) assurant une convergence quasi certaine vers le minimum global. Les résultats de convergence de cette méthode ont ensuite servi de référence pour établir l'intérêt de la nouvelle approche d'optimisation présentée à la section 2 , en terme d'accélération de la convergence.

À partir d'une étude statistique portant sur 100 expériences indépendantes pour chaque fonction test, 
Tableau 1. Taux de réduction du nombre moyen d'évaluations pour la méthode AGA et la méthode de Giannakolou [2] rapporté à un AG classique.

\begin{tabular}{ccc}
\hline & Méth. Giannakoglou & AGA \\
\hline Rastrigin & 2 & 4 \\
Griewank & 6 & 10 \\
\hline
\end{tabular}

la méthode de Giannakoglou et celle proposée (AGA) sont comparées à la méthode des algorithmes génétiques seuls, en fonction du nombre moyen d'évaluations exactes nécessaires à l'obtention d'un niveau de convergence donné $\left(10^{-3}\right)$. Le tableau 1 dresse un comparatif portant sur le taux de réduction du nombre d'évaluations exactes obtenu pour les méthodes hybrides par rapport à la méthode des algorithmes génétiques.

L'algorithme génétique secondaire développé au sein de l'algorithme AGA permet d'améliorer la précision de l'évaluation des individus, et donc la rapidité de convergence vers la solution optimale. L'efficacité de la méthode AGA proposée est ainsi mise en évidence et nous proposons de valider cette approche sur une application d'optimisation aérodynamique 3D automobile.

\section{Application des méthodes d'optimisation en aérodynamique automobile}

Nous appliquons les trois méthodes présentées, à la minimisation du coefficient de traînée d'un véhicule simplifié, dont la forme arrière est paramétrée par trois angles : l'angle $\alpha$ de lunette, l'angle $\beta$ de rétreint et l'angle $\gamma$ de diffuseur $\left((\alpha ; \beta ; \gamma) \in[0,40]^{3}\right.$, voir Fig. 1a). Le coefficient de traînée (ou $C_{x}$ ) est défini par la relation suivante :

$$
C_{x} \equiv C_{x}(\alpha, \beta, \gamma)=\frac{F_{x}}{\frac{1}{2} \rho V_{\infty}^{2} S}
$$

où $\rho$ représente la densité, $S$ la surface apparente, $V_{\infty}$ la vitesse à l'infini et $F_{x}$ la composante longitudinale de la force aérodynamique.

L'écoulement autour de la forme simplifiée est supposé incompressible et stationnaire. La valeur du coefficient de traînée est obtenue à l'aide d'une simulation « rapide * basée sur un logiciel du commerce. Le modèle de turbulence choisi est de type $k-\epsilon$. La veine est modélisée à l'aide de 0,4 million de mailles et la vitesse infinie est de $40 \mathrm{~m} / \mathrm{s}$.

Les différentes méthodes d'optimisation abordées dans cet article (AG et hybrides) sont testées sur ce problème et comparées entre elles. Leur historique de convergence est présenté sur la figure 2 .

L'analyse des résultats montre que les méthodes convergent vers une même valeur finale de $C_{x}$, de l'ordre de 0,117 , en partant d'une même valeur initiale (quasi double) mais en un nombre très différent d'évaluations. Plus précisément, les méthodes hybrides permettent de réduire de façon significative le nombre d'évaluations aérodynamiques (coûteuses en temps) par rapport aux

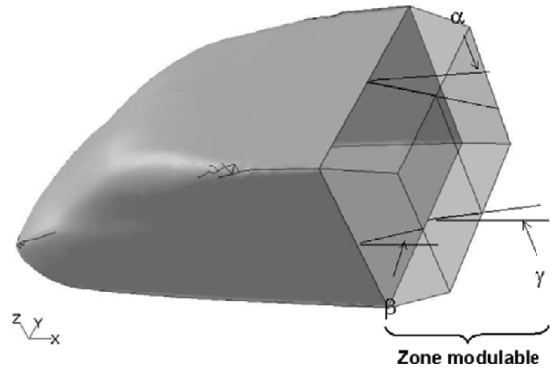

(a) Forme de l'étude

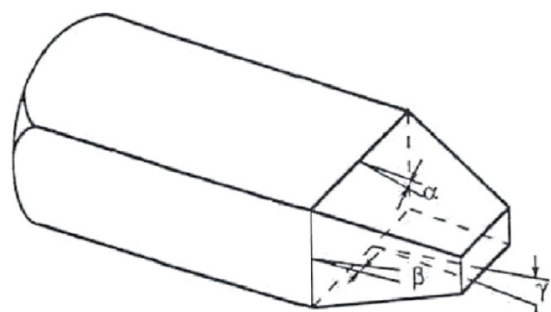

(b) Bluff Body de Sagi et al. [5]

Fig. 1. Forme simplifiée de véhicule, paramétrée par les angles $\alpha, \beta$ et $\gamma$.

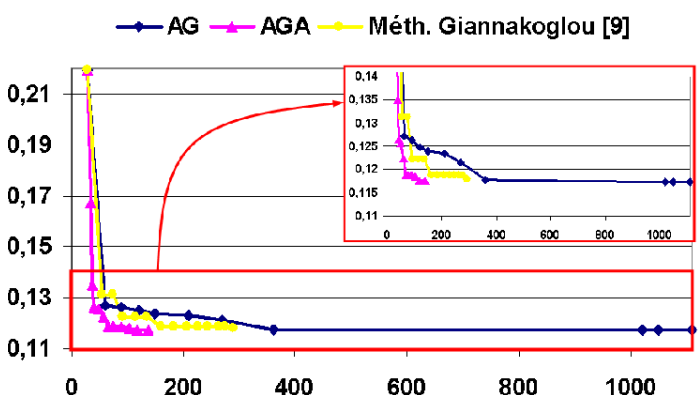

Fig. 2. Historique de convergence pour l'optimisation aérodynamique d'un véhicule simplifié. Cas à 0,4 million de cellules.

algorithmes génétiques : dans un rapport de 7 pour la méthode hybride AGA, et de 3,5 pour celle proposée par Giannakoglou [2]. Afin de valider le potentiel de la méthode AGA, il est nécessaire d'analyser la topologie des écoulements obtenus sur les formes testées. La figure 4 présente une comparaison entre la topologie de l'écoulement arrière de la forme optimisée et celle de la meilleure forme de la population initiale. L'écoulement arrière de la forme optimisée est bien caractéristique d'un véhicule à faible traînée : tore symétrique et faible enveloppe de traînée.

Les angles optimaux obtenus par chacune des méthodes sont très proches du triplet $(24,2 ; 25,1$; 17,7). Or, une étude de Sagi et al. [5], basée sur un développement de Taylor, et appliquée sur un corps simplifié similaire (voir Fig. 1b), montre que la forme arrière optimale est obtenue pour le triplet $(17,8 ; 18,9 ; 9,2)$. Le manque de robustesse de la simulation utilisée lors de notre étude explique la différence obtenue entre les deux géométries optimisées. Il est donc nécessaire d'évaluer chaque forme de véhicule générée par notre méthode, 


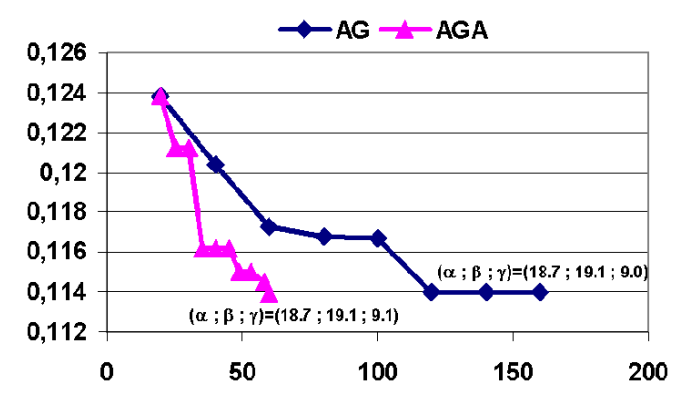

Fig. 3. Historique de convergence pour l'optimisation aérodynamique d'un véhicule simplifié. Cas à 6 millions de cellules.

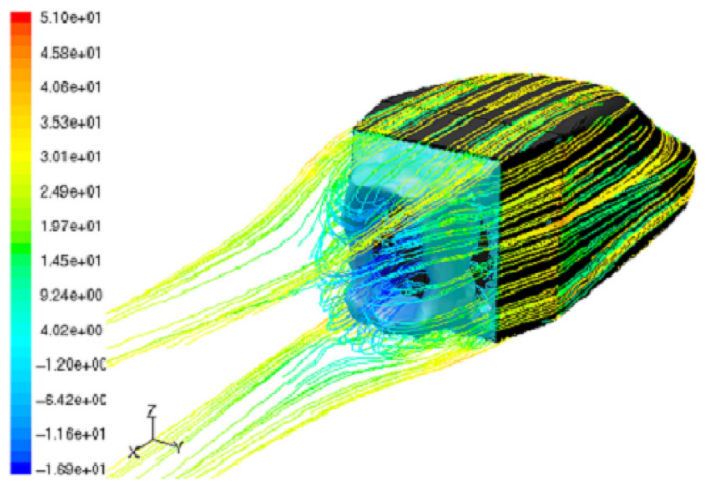

(a)

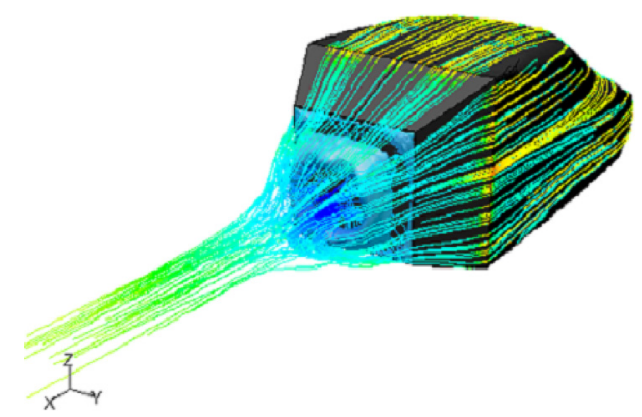

(b)

Fig. 4. Lignes de courant et vitesses longitudinales, forme initiale ((a), $\left.C_{x}=0,22\right)$ et optimisée ((b), $\left.C_{x}=0,117\right)$ Calculs à 0,4 million de cellules.

en se basant sur une modélisation plus précise, mais plus coûteuse en temps de calcul.

La même étude est donc reprise en modifiant, d'une part, les caractéristiques de la modélisation et, d'autre part, l'espace de recherche des trois angles arrières. Le modèle de turbulence est de type RSM, et la veine est modélisée à l'aide de 6 millions de mailles. La vitesse infinie reste à $40 \mathrm{~m} / \mathrm{s}$. L'espace de recherche est restreint à $[15,25] \times[15,25] \times[5,15]$ et seule la méthode hybride AGA est comparée à la méthode des algorithmes génétiques seules. La figure 3 illustre l'évolution du $C_{x}$ des véhicules testés en fonction du nombre de calculs aérodynamiques réalisés. Les angles de la forme «optimale » obtenus, au bout de 60 évaluations, sont alors très proches de ceux obtenus par Sagi et al. (voir Fig. 3). La méthode d'optimisation hybride diminue de moitié le nombre d'évaluations coûteuses en temps, nécessaire pour converger vers la solution «optimale ». L'efficacité de la nouvelle méthode d'optimisation proposée est donc quantifiée et validée sur un cas d'optimisation aérodynamique automobile à 3 paramètres.

\section{Conclusions}

Nous présentons, dans cet article, une nouvelle méthode d'optimisation de forme. Cette méthode est basée sur le couplage d'un algorithme génétique et un réseau de neurones de type RBF. Les paramètres intrinsèques de ce réseau sont déterminés pour chaque interpolation demandée, par un algorithme génétique secondaire. Cette méthode est étudiée, dans un premier temps, sur des cas analytiques, puis sur une application d'optimisation de forme de véhicule simplifié et définie par trois paramètres. Pour chaque étude, une comparaison des performances de plusieurs méthodes (algorithmes génétiques, méthode hybride de type Giannakoklou, et notre méthode hybride) est réalisée.

La méthode de couplage entre les AG et la méthode d'interpolation par réseaux de neurones de type RBF améliore la vitesse de convergence des AG seuls. La réduction du coût de l'optimisation est située entre un facteur 4 et 10 pour les fonctions testées; dans le cas de l'application aérodynamique, une accélération d'un facteur 2 à 7 est obtenue pour l'optimisation de la traînée d'une forme de véhicule simplifié, paramétrée par trois angles.

L'application d'une telle méthode sur des cas véhicules plus complexes est donc prometteuse.

\section{Références}

[1] F. Muyl, L. Dumas, V. Herbert, Hybrid method for aerodynamic shape optimization in automotive industry, Computers and Fluids 33 (2004) 849-858

[2] K.C. Giannakoglou, Designing turbomachinery blades using evolutionary methods, ASME Paper 99-GT-181, 1999

[3] D.E. Goldberg, Genetic Algorithms in Search, Optimization, and Machine Learning, Addison-Wesley, 1989

[4] F. Muyl, Méthode d'optimisation hybride : application à l'optimisation de formes aérodynamiques automobiles, Ph.D. thèse, Université Paris 6, 2003

[5] C.J. Sagi, T. Han, D.C. Hammond, Optimization of Bluff body for minimum drag in ground proximity, AIAA paper, 1992

[6] D.J. Montana, L. Davis, Training feedforward neural network using genetic algorithms, In Proceedings of th 11th International Join Conference on Artificial Intelligence, IJCAI, San Mateo, CA, Morgan-Kaufmann, 1989, pp. 762767 